\title{
Helix-Coil Transition of Parallel-Stranded DNA. Thermodynamics of Hairpin and Linear Duplex Oligonucleotides
}

\author{
Niels B. Ramsing, Karsten Rippe,,$^{\ddagger}$ and Thomas M. Jovin* \\ Department of Molecular Biology, Max Planck Institute for Biophysical Chemistry, Postfach 2841, D-3400 Göttingen, FRG \\ Received April 12, 1989; Revised Manuscript Received July 12, 1989
}

\begin{abstract}
The stabilities have been determined of different DNA double helices constructed with the two constituent strands in a parallel orientation. These molecules incorporate polarity-inverting loop structures (hairpins) or nucleotide sequences (duplexes) which impose the desired polarity on the two strands constituting the sugar-phosphate backbone. The hairpins consisted of $\mathrm{d}(\mathrm{A} \cdot \mathrm{T})_{n}$ stems $(n=8$ or 10$)$ and either a $5^{\prime}-\mathrm{p}-5^{\prime}$ linkage in a d(C) ${ }_{4}$ loop (ps-C8 and ps-C10) or a $3^{\prime}-\mathrm{p}-3^{\prime}$ linkage in a $\mathrm{d}(\mathrm{G})_{4}$ loop (ps-G10). The linear duplexes had 21-nt (ps-C2-C3) and 25-nt (ps-D1·D2, ps-D3-D4) mixed A,T sequences and normal chemical linkages throughout. Reference molecules with normal antiparallel helical orientations (hairpins aps-C8, aps-C10, and aps-G10 and duplexes aps-C3.C7, aps-D1·D3, and aps-D2.D4) were also synthesized and studied. Hydrogen bonding in ps-DNA is via reverse Watson-Crick base pairs, and the various constructs display spectroscopic, chemical, biochemical, and electrophoretic properties distinct from those of their aps counterparts. For example, both the ps and aps molecules show a pronounced UV absorption hyperchromicity upon melting, but the spectral distribution is not the same. Thus, the difference spectra (ps - aps) in the native state are characterized by a positive peak at $252 \mathrm{~nm}$, an isosbestic point at $267 \mathrm{~nm}$, and a negative peak at $282 \mathrm{~nm}$. Temperature-dependent absorbances were recorded at selected wavelengths and in the form of complete spectra to derive the thermodynamic parameters for the helix-coil transitions. The relative absorption changes as well as the thermodynamic parameters are in accordance with an intramolecular helix-coil transition for the hairpins and a bimolecular transition for the linear duplexes. There is no indication of higher order structures such as dimers and concatamers. The van't Hoff melting enthalpies $\Delta H_{\mathrm{vH}}$ are found to be independent of salt concentration and about $20 \%$ lower in the case of the ps-DNAs. The latter also exhibit lower melting temperatures, $T_{\mathrm{m}}$, by about 10 and $15^{\circ} \mathrm{C}$ for the hairpins and duplexes, respectively. However, all the DNAs show a similar dependency upon salt concentration $\left(\partial T_{\mathrm{m}} / \partial \log [\mathrm{NaCl}]=17 \pm\right.$ $3{ }^{\circ} \mathrm{C}$ ). Parallel-stranded DNA is second only to B-DNA in helical stability under physiological salt conditions and temperature.
\end{abstract}

$\mathbf{P}_{a}$ Dstranded DNA (ps-DNA) ${ }^{1}$ is a novel doublestranded helical form of DNA characterized by two major features (Pattabiraman, 1986; van de Sande et al., 1988). (1) The two sugar-phosphate backbones are oriented in the same, i.e. parallel, orientation. (2) The secondary structure is established by reverse Watson-Crick base pairs between the $\mathrm{A}$ and $\mathrm{T}$ bases constituting the sequences examined to date. We and our colleagues have recently described (van de Sande et al., 1988; Ramsing \& Jovin, 1988; Germann et al., 1988; Rippe et al., 1989) a series of molecules designed to test the predictions from force field calculations by Pattabiraman (1986) of a stable structure for the homopolymeric duplex $\mathrm{d}(\mathrm{A})_{6} \cdot \mathrm{d}(\mathrm{T})_{6}$ with the features given above. The energy calculations indicated that the stabilities of the proposed conformation and that of the corresponding conventional antiparallel B-DNA helix would be similar. In the first test of the existence of parallel-stranded (ps) DNA, a series of hairpin molecules were synthesized in which $5^{\prime}-\mathrm{p}-5^{\prime}$ and $3^{\prime}-\mathrm{p}-3^{\prime}$ phosphodiester bonds were introduced in the loops consisting of four $\mathrm{C}$ nucleotides (hairpin ps-C10) or four $\mathrm{G}$ nucleotides (hairpin ps-G10). This was done to restrict the decanucleotide $\mathrm{d}(\mathrm{A})_{10^{\circ}} \mathrm{d}(\mathrm{T})_{10}$ stems to a parallel orientation (van de Sande et al., 1988). We found (van de Sande et al., 1988) that (i) the ps hairpins are only slightly less stable than reference aps molecules and that (ii) they exhibit characteristic differences

\footnotetext{
* To whom correspondence should be addressed.

‡ Work done as part of the Diplom degree, awarded by the University of Göttingen, FRG.
}

in absorption and CD spectra, enzyme substrate specificities, and drug-binding properties. These findings were extended to duplexes of oligonucleotides with sequences designed such that the parallel orientation was greatly favored over the alternative antiparallel modes of interaction (Ramsing \& Jovin, 1988; Germann et al., 1988; Rippe et al., 1989). In this paper, we document in detail the thermodynamic properties of psDNA on the basis of the helix-coil transitions of the molecular species we have examined to date.

\section{Materials ANd Methods}

Oligonucleotide Synthesis and Characterization. Oligonucleotides ps-C8, ps-C10, ps-G10, aps-C8, aps-C10, and aps-G10 (Figure 1A) were synthesized by modifications of phosphoramidite chemistry as described elsewhere (van de Sande et al., 1988) and were supplied by Dr. van de Sande. They were purified by high-performance liquid chromatography on a poly(styrene divinylbenzene) resin (PRP-1) and by preparative gel electrophoresis (Germann et al., 1987). For spectral measurements they were further purified by gel filtration on a Bio-Rad P-6DG column. The duplexes ps-C2.C3 and aps-C3.C7 (Figure 1B) were prepared according to the method of Ramsing and Jovin (1988) and the duplexes ps-

\footnotetext{
${ }^{1}$ Abbreviations: nt, nucleotide; ps, parallel-stranded; aps, antiparallel-stranded; ps-DNA, parallel-stranded DNA; aps-DNA, antiparallelstranded DNA; duplex (used alone), double-stranded helical DNA (hairpins or linear molecules)
} 
ps-C8 (3'-3', C-loop, 8 bp stem)

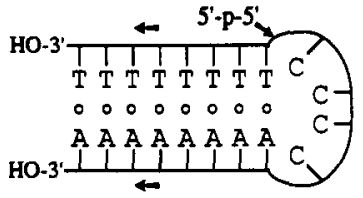

ps-C10 (3'-3', C-loop, 10 bp stem)

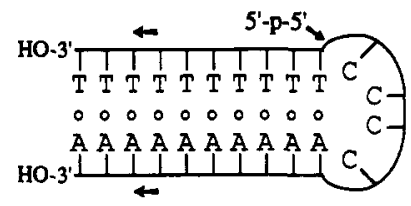

ps-G10 (5'-5', G-loop, 10 bp stem)

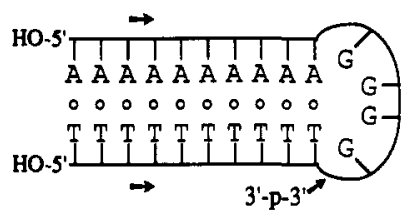

aps-C8 (5'-3', C-loop, 8 bp stem)

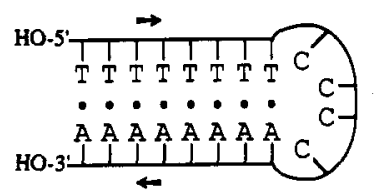

aps-C10 (5'-3', C-loop, 10 bp stem)

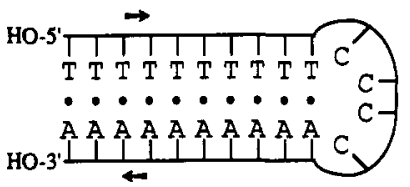

aps-G10 (5'-3', G-loop, 10 bp stem)

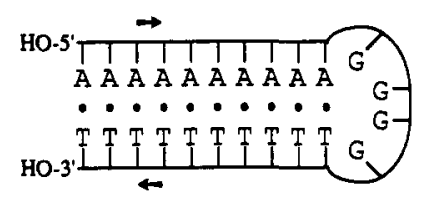

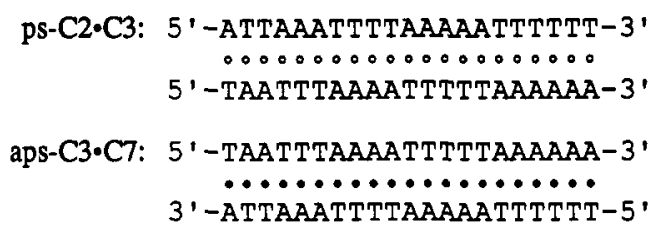

C

ps-D1·D2: 5' -AAAAAAAAAATAATTTTAAATATTT - 3'

०००00000000000000000000

5 '-TTTTTTTTTTATTAAAATTTATAAA-3'

ps-D3·D4: 5 ' -AAATATTTAAAATTATTTTTTTTTT-3'

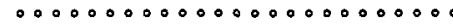

5 ' -TTTATAAATTTTAATAAAAAAAAAA-3'

aps-D1 •D3: 5 '-AAAAAAAAAATAATTTTAAATATTT-3'

...................

3 '-TTTTTTTTTATTAAAATTATAAA-5'

aps-D2.D4: 5 '-TTTTTTTTTTATTAAAATTTATAAA-3'

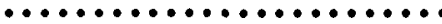

$3^{\prime}$-AAAAAAAAAATAATTTTAAATATTT-5'

FIGURE 1: Oligonucleotide duplexes analyzed in this study. The unusual phosphodiester bonds $\left(5^{\prime}-\mathrm{p}-5^{\prime}\right.$ and $\left.3^{\prime}-\mathrm{p}-3^{\prime}\right)$ in the ps hairpins are indicated. The Watson-Crick base pairs of the antiparallel stem regions are shown by the symbol 1 and the reverse Watson-Crick base pairs of the parallel-stranded duplex by the symbol O. (A) Hairpin structures (van de Sande et al., 1988). (B) C-series duplexes (Ramsing \& Jovin, 1989). (C) D-series duplexes (Rippe et al., 1989).

D1·D2, ps-D3·D4, aps-D1·D3, and aps-D2·D4 (Figure 1C) according to the method of Rippe et al. (1989). All solutions contained a standard buffer, $10 \mathrm{mM}$ sodium cacodylate, $\mathrm{pH}$ 7.2 , in addition to the specified salts $\left(\mathrm{NaCl}, \mathrm{MgCl}_{2}\right)$. The molar extinction coefficients of the oligonucleotides (Table I) were determined by spectral and phosphate analysis (van de Sande et al., 1988; Ramsing \& Jovin, 1988; Rippe et al., 1989). The DNA duplexes were prepared for measurements by mixing the appropriate oligonucleotides in the desired salt solutions, heating to $50-60^{\circ} \mathrm{C}$ for $5 \mathrm{~min}$, and cooling slowly to room temperature.

Spectroscopic Measurements and Thermal Transitions. Ultraviolet absorption spectra were recorded on a Uvikon 820 spectrophotometer equipped with thermostated cuvette holders. Measurements were made in four optically matched $4 \mathrm{~mm}$ (width) $\times 10 \mathrm{~mm}$ (path length) quartz cuvettes, each with a sample volume of $1 \mathrm{~mL}$.

The thermal transitions were determined on the same instrument controlled by a Tandy TRS- 80 microcomputer and associated electronics. The data were transferred to a VAX 8600 for further processing and analysis. Each experiment involved the recording of absorbances at discrete wavelengths for all cuvettes as well as the temperature in an additional cuvette located in the same holder. The temperature was then raised by an increment $\Delta T$, and new absorption measurements were made after equilibrium was reached at the new temperature. Two different protocols were used, yielding equivalent results. According to the first protocol the absorbance at three different wavelengths, 250,267 , and $279 \mathrm{~nm}$, were recorded in the temperature range $12-75^{\circ} \mathrm{C}$, with 200 data points for an initial phase of increasing temperatures and another 200 during a subsequent cooling phase. The heating rate was approximately $0.2{ }^{\circ} \mathrm{C} / \mathrm{min}$. The second protocol recorded whole digitized spectra, 220-320 $\mathrm{nm}$ in steps of 2 $\mathrm{nm}$, at $4^{\circ} \mathrm{C}$ intervals during both heating and cooling cycles.
Thermal equilibration was attained in $2 \mathrm{~min}$, and a further 4 min was allowed to establish chemical equilibrium. Spectra were obtained in the temperature range $2-98^{\circ} \mathrm{C}$, but only values below $85^{\circ} \mathrm{C}$ were used in the subsequent analysis.

The absorbances of each sample at each temperature and wavelength were corrected for cuvette and solvent contributions by subtracting the smoothed absorbance of a reference cuvette included in each experiment. The data were subsequently adjusted for any systematic differences between reference and sample cuvettes (which were wavelength independent for the matched set of cuvettes) by subtracting the average absorbance above $305 \mathrm{~nm}$ at each temperature. All absorbances were finally scaled to $4{ }^{\circ} \mathrm{C}$ with respect to thermal expansion of water, ignoring, however, the influence of salt. The $\mathrm{NaCl}$ concentrations of selected samples were checked by measuring density and refractive index. The 3-D spectral surfaces in Figure 3 were plotted with the ICEX GRAFkit graphics program running on a DEC MicroVax II computer.

Analysis of Transition Data. The corrected data from the thermal transition experiment were analyzed according to a concerted two-state model for the helix to coil transition as described under Results and Discussion. The measured experimental quantity was $A_{\lambda}$, the absorbance at wavelength $\lambda$ as a function of temperature. A data set consisted of a family of $A_{\lambda}(T)$ curves. The extinction coefficients in both the helical and the random coiled state were assumed to be linear functions of temperature according to

$$
A_{\lambda}=\left[\epsilon_{0}{ }^{\mathrm{h}}\left(1+\beta^{\mathrm{h}} T\right)(1-\alpha)+\epsilon_{0}^{\mathrm{c}}\left(1+\beta^{\mathrm{c}} T\right) \alpha\right] n C_{\mathrm{t}}
$$

in which the superscripts $h$ and $c$ of the molar (base units) extinction coefficients, $\epsilon_{0}$, refer to the helical and coil forms respectively, $\beta$ is the linear temperature dependence coefficient of $\epsilon_{0}, \alpha$ is the fractional degree of transition to the coil form which depends on $T_{\mathrm{m}}$ and $\Delta H_{\mathrm{vH}}$ (eq 6,9, and 10), $n$ is the oligonucleotide chain length, and $C_{\mathrm{t}}$ is the total concentration 

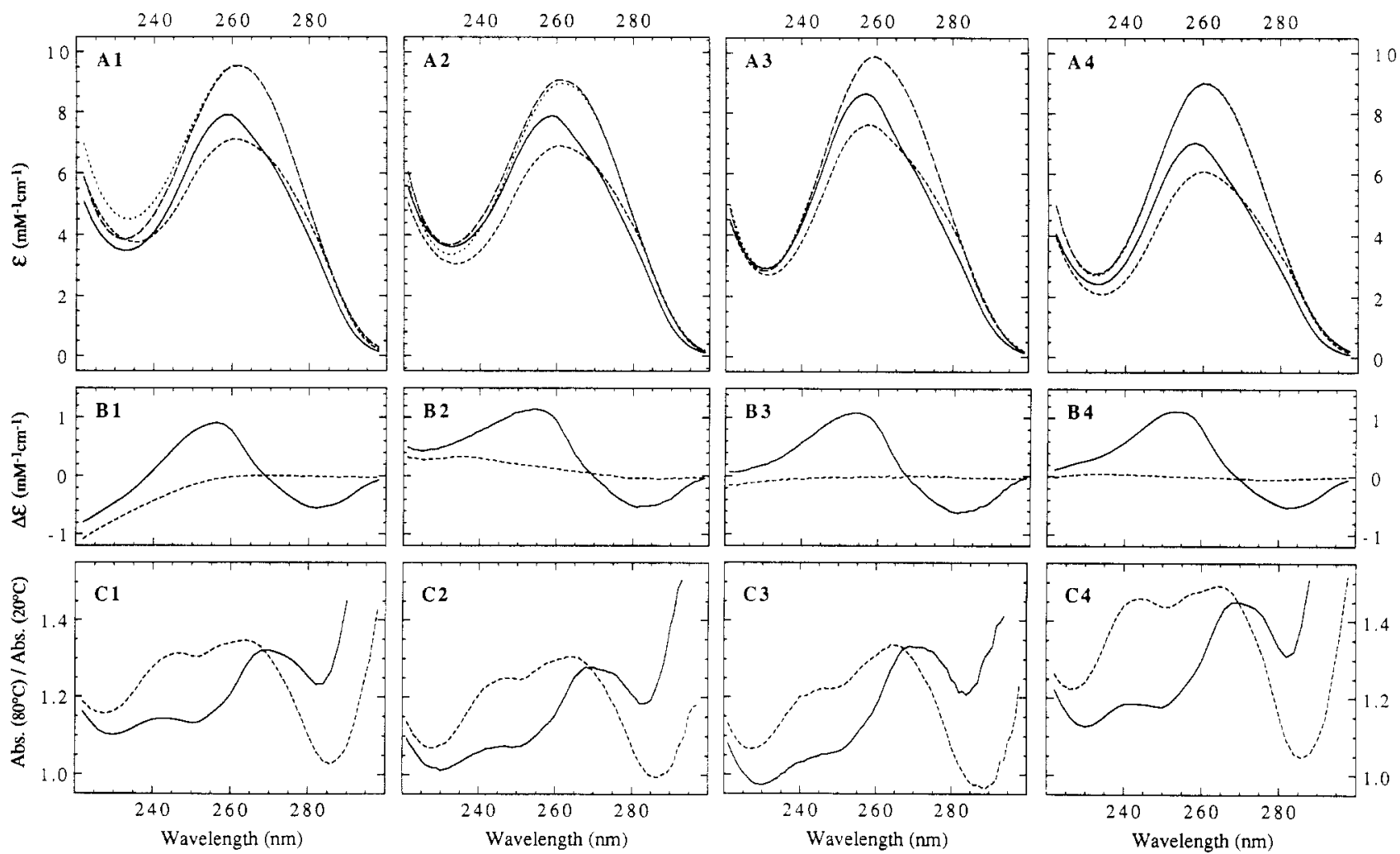

FIGURE 2: Ultraviolet absorption spectra of ps and aps oligonucleotides. (A1, B1, C1) ps-C8 and aps-C8 hairpins. (A2, B2, C2) ps-C10 and aps-C10 hairpins. (A3, B3, C3) ps-G10 and aps-G10 hairpins. (A4, B4, C4) ps-C2.C 3 and aps-C $3 \cdot \mathrm{C} 7$ linear duplexes, all in $0.1 \mathrm{M} \mathrm{NaCl}$ and $10 \mathrm{mM}$ sodium cacodylate, $\mathrm{pH} 7.2$. (A1-4) UV spectra at $20^{\circ} \mathrm{C}$, ps duplexes (-) and aps duplexes (--), and at $80^{\circ} \mathrm{C}$, melted ps duplexes $(--)$ and melted aps duplexes (*.). (B1-4) Difference spectra (ps - aps) at $20^{\circ} \mathrm{C}(-)$ and at $80^{\circ} \mathrm{C}(-\cdots)$. (C1-4) Hyperchromicity spectra of ps duplexes (-) and aps duplexes (-), expressed as the ratio of absorbances at 80 and $20^{\circ} \mathrm{C}$.

of oligonucleotide strands. In the case of two coil species, $\epsilon_{0}{ }^{\mathrm{C}}$ is the mean of the two extinction coefficients.

The experimental data were computer analyzed by a nonlinear regression to eq $1,6,9$, and 10 . Initial parameters for each wavelength were found by dividing the transition curves in three parts. The linear parameters $\epsilon_{0}^{\mathrm{h}}, \beta^{\mathrm{h}}, \epsilon_{0}^{\mathrm{c}}$, and $\beta^{\mathrm{c}}$ were estimated for the two linear segments. $T_{\mathrm{m}}$ and $\Delta H_{\mathrm{vH}}$ were determined from the central part of each curve and then from a global analysis of the complete data set restricted to spectral regions exhibiting $>30 \%$ hyperchromicity upon denaturation. The initial parameters were improved by two nonlinear least-squares minimization routines, both from the IMSL library of mathematical FORTRAN subroutines. One procedure (DBCLSJ) is a Levenberg-Marquardt type that uses gradient information from the analytical Jacobian matrix. It is fast but does not change parameters that have a low partial derivative. The other procedure (DBCPOL) is a simplex type that moves over the $n$-dimensional parameter surface. It changes all parameters but converges slowly. These two routines were used in alternation and with different initial step sizes to avoid local minima.

Analysis of individual transition curves showed $T_{\mathrm{m}}$ and $\Delta H_{\mathrm{vH}}$ to be independent of wavelength. [One exception was the duplex ps-C2.C3 with which the effect of intermediate species and/or alternative conformations appeared to reduce the validity of the two-state model (Ramsing \& Jovin, 1988).] Therefore, we used the results of the single-wavelength analysis as initial parameters in a global multiwavelength analysis, fitting all the data simultaneously such that the linear parameters were assumed to be wavelength dependent, whereas $T_{\mathrm{m}}$ and $\Delta H_{\mathrm{vH}}$ were universal variables applicable to all wavelengths (Ramsing \& Jovin, 1988). In the case of experiments using the first protocol, and heating only to $75^{\circ} \mathrm{C}$, we found the values to be independent of direction (increasing versus decreasing temperatures), and thus included both slopes in the global analysis. In the case of experiments using the second protocol, we analyzed each direction separately and restricted the data set to wavelengths displaying a total absorbance increase of more than $30 \%$ and to a selected temperature range around the melting temperature. The second limitation was imposed because the assumption that the extinction coefficients are linear functions of temperature is valid only over a limited temperature range. The latter depends upon the observed cooperativity, as reflected in $\Delta H_{\mathrm{vH}}$, and was typically $T_{\mathrm{m}} \pm 30^{\circ} \mathrm{C}$ in our experiments. The analyses were performed with and without a requirement that the linear parameters be positive. This constraint was useful for melting temperatures close to either end of the experimental temperature range. In those cases, the linear extinction parameters were largely undefined and had to be confined to positive values to ensure convergence.

The statistical significance of the analysis results were investigated by using the program StatView SE+ (Abacus Concepts, Inc.) to (1) determine $95 \%$ confidence limits for the average values reported in the tables, (2) test the statistical significance of differences between data obtained with different experimental protocols by using a paired $t$-test and the differences between mean values by using an unpaired $t$-test, and (3) determine the linear regression lines and confidence limits for slopes and intercepts.

\section{RESULTS AND DISCUSSION}

Spectroscopic Properties of the ps Hairpins and Linear Duplexes. The ultraviolet absorption spectra of all parallelstranded DNAs examined in both $\mathrm{NaCl}$ and $\mathrm{MgCl}_{2}$ solutions 


\begin{tabular}{|c|c|c|c|c|c|c|c|c|}
\hline \multirow[b]{2}{*}{ DNA duplex } & \multicolumn{3}{|c|}{$\begin{array}{l}\text { extinction coefficients }{ }^{b} \\
\text { in } 0.1 \mathrm{M} \mathrm{NaCl}, 20^{\circ} \mathrm{C}\end{array}$} & \multicolumn{3}{|c|}{ absorbance ratios in $0.1 \mathrm{M} \mathrm{NaCl}, 20^{\circ} \mathrm{C}$} & \multicolumn{2}{|c|}{$\begin{array}{l}A_{250} / A_{284} \text { in } \\
0.1 \mathrm{M} \mathrm{NaCl}\end{array}$} \\
\hline & $\overline{\lambda_{\max }}$ & $\epsilon_{\max }$ & $\overline{\epsilon_{260}}$ & $\overline{A_{250} / A_{260}}$ & $A_{280} / A_{260}$ & $A_{290} / A_{260}$ & $20^{\circ} \mathrm{C}$ & $80^{\circ} \mathrm{C}$ \\
\hline \multicolumn{9}{|c|}{ parallel-stranded duplexes } \\
\hline $\mathrm{ps}-\mathrm{C} 8$ & 258 & 7.9 & 7.9 & 0.83 & 0.49 & 0.13 & 2.4 & 2.2 \\
\hline ps-C 10 & 259 & 7.9 & 7.8 & 0.85 & 0.48 & 0.12 & 2.5 & 2.3 \\
\hline ps-G10 & 257 & 8.7 & 8.4 & 0.92 & 0.43 & 0.13 & 3.0 & 2.6 \\
\hline $\mathrm{ps}-\mathrm{C} 2 \cdot \mathrm{C} 3$ & 258 & 7.0 & 6.9 & 0.85 & 0.42 & 0.09 & 3.1 & 2.7 \\
\hline ps-D1.D2 & 258 & 7.0 & 7.0 & 0.84 & 0.41 & 0.08 & 3.2 & 2.7 \\
\hline ps-D3.D4 & 258 & 7.1 & 7.0 & 0.84 & 0.40 & 0.08 & 3.3 & 2.7 \\
\hline \multicolumn{9}{|c|}{ antiparallel-stranded duplexes } \\
\hline aps-C8 & 260 & 7.1 & 7.1 & 0.82 & 0.62 & 0.20 & 1.8 & 2.3 \\
\hline aps-C10 & 261 & 6.9 & 6.9 & 0.80 & 0.62 & 0.20 & 1.8 & 2.2 \\
\hline aps-G10 & 258 & 7.6 & 7.6 & 0.89 & 0.56 & 0.19 & 2.1 & 2.6 \\
\hline aps-C3.C7 & 260 & 6.1 & 6.1 & 0.79 & 0.55 & 0.16 & 2.0 & 2.7 \\
\hline aps-D1.D3 & 260 & 6.0 & 6.0 & 0.77 & 0.56 & 0.16 & 2.0 & 2.8 \\
\hline aps-D2.D4 & 260 & 6.1 & 6.1 & 0.78 & 0.54 & 0.16 & 2.0 & 2.7 \\
\hline
\end{tabular}

${ }^{a}$ The subscripts refer to wavelengths in nanometers. All values were measured in $10 \mathrm{mM}$ sodium cacodylate, $\mathrm{pH} 7.2$. The $\mathrm{Na}^{+}$concentration including the buffer contribution is $0.11 \mathrm{M} .{ }^{b} \lambda_{\max }$ is the wavelength in nanometers with the highest extinction coefficient; $\epsilon_{\max }$ and $\epsilon_{260}$ are in units of $\mathrm{mM}^{-1} \mathrm{~cm}^{-1}$.
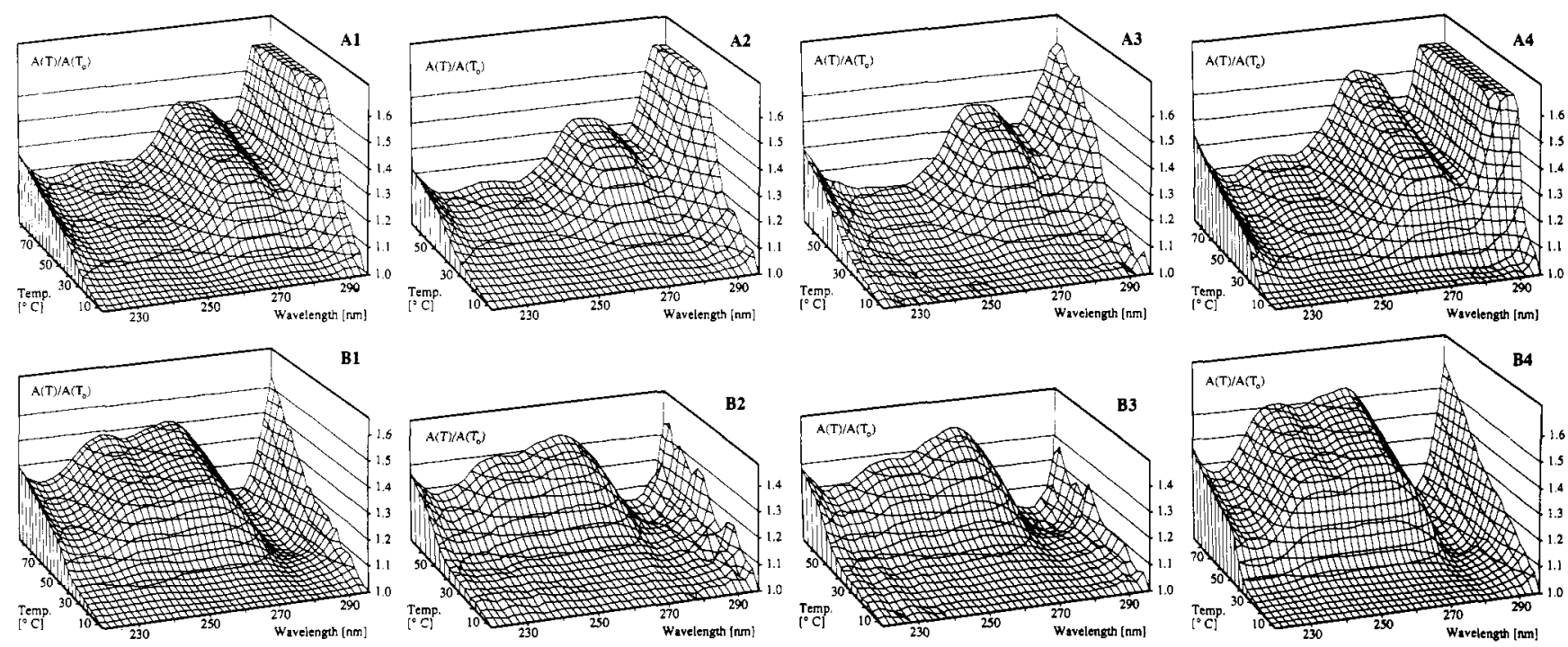

FIGURE 3: Thermally resolved ultraviolet absorption spectra of ps and aps helices expressed as the hyperchromicity, the ratio $A_{\lambda}(T) / A_{\lambda}\left(T_{0}\right)$, where $T_{0}$ is the initial temperature. (Top row) Parallel-stranded DNAs (A1) ps-C8, (A2) ps-C10, (A3) ps-G10, and (A4) ps-C2.C3. (Bottom row) Antiparallel-stranded DNAs (B1) aps-C8, (B2) aps-C10, (B3) aps-G10, and (B4) aps-C3.C7. All are in $0.1 \mathrm{M} \mathrm{NaCl}$ and $10 \mathrm{mM}$ sodium cacodylate, $\mathrm{pH} 7.2$.

differ in a characteristic manner from those of the corresponding antiparallel B-DNA species (Figure 2, A1-4; Table I; van de Sande et al., 1988; Ramsing \& Jovin, 1988; Germann et al., 1988; Rippe et al., 1989). The difference spectra (ps - aps, Figure 2, B1-4) have a positive peak at 250-255 nm, a negative peak at $280-285 \mathrm{~nm}$, and an isosbestic point at 270 $\mathrm{nm}$. This blue shift in the ultraviolet absorption spectra is unique for ps-DNA and indicative of changes in the electronic structure and stacking geometries of the respective helices. The ultraviolet absorption spectra under denaturing conditions (80 ${ }^{\circ} \mathrm{C}$ ) are virtually identical for oligonucleotides of the same or isomeric nucleotide sequence, even for the hairpins containing a variant phosphodiester bond with a $3^{\prime}-\mathrm{p}-3^{\prime}$ or $5^{\prime}-\mathrm{p}-5^{\prime}$ linkage (Figure 2, Al-4; van de Sande et al., 1988). It follows that the relative absorption increase upon helix-coil transition (Figure 2, $\mathrm{C} 1-4$ ) displays a strong wavelength dependence which is markedly different in the case of ps-DNA as compared to aps-DNA. This qualitative difference provides one of the easiest ways to distinguish between ps-DNA and apsDNA. A characteristic value is the absorbance ratio $A_{250} / A_{284}$, which decreases upon melting of ps-DNA but increases upon melting of aps-DNA (Table I). For the species exclusively composed of A.T base pairs, the ratio $A_{250} / A_{284}$ at $20^{\circ} \mathrm{C}$ is $3.2 \pm 0.1$ and $2.0 \pm 0.1$ for $\mathrm{ps}$ and aps duplexes, respectively. The spectral data are summarized in Table I.

Wavelength-Dependent Hyperchromicity of the ps and aps Duplexes. The thermally induced hyperchromicity in the ultraviolet absorption spectrum exhibited by ps and aps duplexes is indicative of cooperative transitions from base-stacked double-stranded structures to a melted random coiled state. The thermal transitions were recorded as a set of absorption spectra obtained at discrete temperature intervals in both the forward and reverse directions. Examples are given in Figure 3 in the form of hyperchromicity plots, in which the absorbances are normalized to the values for each wavelength at the initial (low) temperature, a useful representation for distinguishing between different DNA conformations (N. B. Ramsing and T. M. Jovin, unpublished data). From experiments conducted to date with secondary structures consisting exclusively of A.T base pairs (van de Sande et al., 1988; Ramsing \& Jovin, 1988), these spectral properties (i) do not appear to depend appreciably on the precise sequence and (ii) are the same for both hairpins and linear duplexes. That is, they reflect primarily the overall helical geometry. 
The hyperchromicity spectra in Figure 3 of ps and aps helices consisting exclusively of A.T base pairs are distinctly different. Whereas both ps hairpins (Figure 3, A1-3) and linear ps duplexes (Figure 3, A4) are characterized by a trough at $230 \mathrm{~nm}$ and a crest near $270 \mathrm{~nm}$, the corresponding aps molecules (Figure 3, B1-4) show a plateau in the range of 240-270 nm and a strong cleft at $285 \mathrm{~nm}$. The peak hyperchromicity (ca. 1.5) of the linear duplexes was greater than that of the hairpins, presumably due to the relatively temperature independent contributions from the $\mathrm{d}(\mathrm{C})_{4}$ or $\mathrm{d}(\mathrm{G})_{4}$ loops (van de Sande et al., 1988) in the latter case.

Helix-Coil Transition Models. The helix-coil transitions for the hairpin, an intramolecular process, and for the heteroduplex molecules, an intermolecular process, can be represented according to the formalism for a concerted two-state model (Marky \& Breslauer, 1987)

intramolecular transition (hairpins)

$$
\mathrm{C}^{\mathrm{h}} \Leftrightarrow \mathrm{C}^{\mathrm{c}}
$$

intermolecular transition (linear duplexes)

$$
\mathrm{C}_{i} \cdot \mathrm{C}_{j} \Leftrightarrow \mathrm{C}_{i}+\mathrm{C}_{j}
$$

in which $\mathrm{C}^{\mathrm{h}}$ and $\mathrm{C}^{\mathrm{c}}$ are the base-paired (helical stem) and coil forms of the hairpins, respectively, and the two complementary strands constituting the duplex molecules are represented by $\mathrm{C}_{i}$ and $\mathrm{C}_{j}$. The equilibrium constant, $K$, is given by

hairpin transition

$$
K=\left[\mathrm{C}^{\mathrm{c}}\right] /\left[\mathrm{C}^{\mathrm{h}}\right]=\alpha /(1-\alpha)
$$

linear duplex transition

$$
K=\left[\mathrm{C}_{i}\right]\left[\mathrm{C}_{j}\right] /\left[\mathrm{C}_{i} \mathrm{C}_{j}\right]=\alpha^{2} /(2(1-\alpha)) \mathrm{C}_{\mathrm{t}}
$$

where $\alpha$ is the fractional degree of transition to the coil form and $C_{1}$ the total oligonucleotide strand concentration. The van't Hoff enthalpy $\Delta H_{\mathrm{vH}}$ for the concerted, cooperative thermal transition is defined by

$$
\begin{aligned}
\Delta H_{\mathrm{vH}}=-R\left(\frac{\partial \ln K}{\partial(1 / T)}\right) & \Rightarrow \\
\frac{K_{1 / 2}}{K} & =\exp \left[\frac{\Delta H_{\mathrm{vH}}}{R}\left(\frac{1}{T}-\frac{1}{T_{\mathrm{m}}}\right)\right] \equiv E
\end{aligned}
$$

where $R$ is the gas constant, $T$ the absolute temperature, and $T_{\mathrm{m}}$ the melting temperature defined as the midpoint of duplex dissociation $(\alpha=1 / 2) . K_{1 / 2}$, the equilibrium constant at the melting temperature, is found from eq 4 and 5 as

hairpin transition

$$
K_{1 / 2}=1
$$

linear duplex transition

$$
K_{1 / 2}=C_{t} / 4
$$

Solving for $\alpha$ in eq 4 and 5 using the appropriate combination of eq 6-8, we obtain $\alpha$ as a function of $T_{\mathrm{m}}$ and $\Delta H_{\mathrm{vH}}$ for each of the models

hairpin transition

$$
\alpha=1 /(1+E)
$$

linear duplex transition

$$
\alpha=\left[(1+8 E)^{1 / 2}-1\right] / 4 E
$$

The transition entropy $\Delta S$ was calculated from the Gibbs equation

$$
\Delta G=-R T \ln K=\Delta H-T \Delta S
$$

where $\Delta G$ is the Gibbs free energy of transition. At the melting point, we obtain from eq 7,8 , and 11

hairpin transition

$$
\Delta S=\Delta H_{\mathrm{vH}} / T_{\mathrm{m}}
$$

linear duplex transition

$$
\Delta S=\Delta H_{\mathrm{vH}} / T_{\mathrm{m}}+R \ln \left(C_{\mathrm{t}} / 4\right)
$$

The melting temperature for the hairpin transition is independent of strand concentration, whereas for the duplex transition (eq 3) $T_{\mathrm{m}}$ depends upon $C_{\mathrm{t}}$.

$$
\frac{1}{T_{\mathrm{m}}}=\frac{\Delta S}{\Delta H_{\mathrm{vH}}}-\frac{R}{\Delta H_{\mathrm{vH}}} \ln \left(\frac{C_{\mathrm{t}}}{4}\right)
$$

Thermodynamics of the Helix to Coil Transition of ps-and aps-DNA. All species demonstrated well-defined, fully reversible thermal transitions. In solutions containing $\mathrm{MgCl}_{2}$ $(0.5-8 \mathrm{mM})$ or $\mathrm{NaCl}(0.05-1.0 \mathrm{M})$, very good fits to the two-state model were achieved, both from the sets of $A_{\lambda}$ vs $T$ slices and from global analyses. The experimental data (Figure 4A) could be reconstructed with very high precision (Figure 4B) from the computed linear parameters and the universal values for $T_{\mathrm{m}}$ and $\Delta H_{\mathrm{vH}}$, as indicated by the surface generated as the residuals between the experimental and calculated absorbances (Figure 4C) and by the superposed $A_{\lambda}$ vs $T$ profiles (Figure 4D). Both $T_{\mathrm{m}}$ and $\Delta H_{\mathrm{vH}}$ are lower for ps- than for aps-DNA, as shown in Figure 4D for ps-C10 and aps-C10.

Deviations from a simple behavior were observed in some of the experiments with ps- $\mathrm{C} 2 \cdot \mathrm{C} 3$, as evidenced by greater differences in the computed $\Delta H_{\mathrm{vH}}$ at different wavelengths and salt concentrations (Table II). We attribute this phenomenon to the existence of competing equilibria involving finite concentrations of antiparallel homoduplexes and heteroduplexes. This behavior was not observed for the more highly optimized D series of oligonucleotides (Rippe et al., 1989) nor for the various hairpins.

The thermodynamic parameters derived from the analysis are summarized in Table II. The values obtained for $\Delta H_{\mathrm{vH}}$ were independent of the direction (ascending or descending) of the thermal transition, and values from analyses of both slopes are included in the table. $\Delta H_{\mathrm{vH}}$ was also independent of ion concentration for $\mathrm{NaCl}$ concentrations in the range $0.1-1.0 \mathrm{M}$ as well as for $\mathrm{MgCl}_{2}$ concentrations from 0.5 to $8 \mathrm{mM}$. An example is shown in Figure $5 \mathrm{~B}$ for ps-C10, the curved lines being $95 \%$ confidence limits of $\Delta H_{\mathrm{vH}}$ as a function of $\mathrm{Na}^{+}$concentration. $\Delta H_{\mathrm{vH}}$ was consistently lower (about $20 \%$ ) for the ps compared to the aps helix.

$\mathrm{Mg}^{2+}$ exerts a strongly stabilizing effect on both ps and aps helices, even at low concentration (less than $2 \mathrm{mM}$ ) as is shown by the increase of $\Delta H_{\mathrm{vH}}$ and $\Delta G$ (Table II). The stabilization appears to be more pronounced for the parallel-stranded helices as the $T_{\mathrm{m}}$ difference (aps - ps) is smaller in $2 \mathrm{mM} \mathrm{MgCl}$ than in $0.1 \mathrm{M} \mathrm{NaCl}$ (Table III). In the presence of $2 \mathrm{mM} \mathrm{MgCl}_{2}$, added $\mathrm{NaCl}$ produced only a slight destabilizing effect on the linear duplexes, in accordance with prior data for helix-coil transitions of B-DNA at $\mathrm{Mg}^{2+}$ phosphate ratios $>0.5$ (Record, 1975).

The enthalpy change per mole of nearest-neighbor interaction, $\Delta h$

$$
\Delta h=\Delta H_{\mathrm{vH}} /(n-1)
$$

is depicted for all species in both $\mathrm{Na}^{+}$(Figure 6A) and $\mathrm{Mg}^{2+}$ (Figure 6B), with $95 \%$ confidence limits as error bars. The 

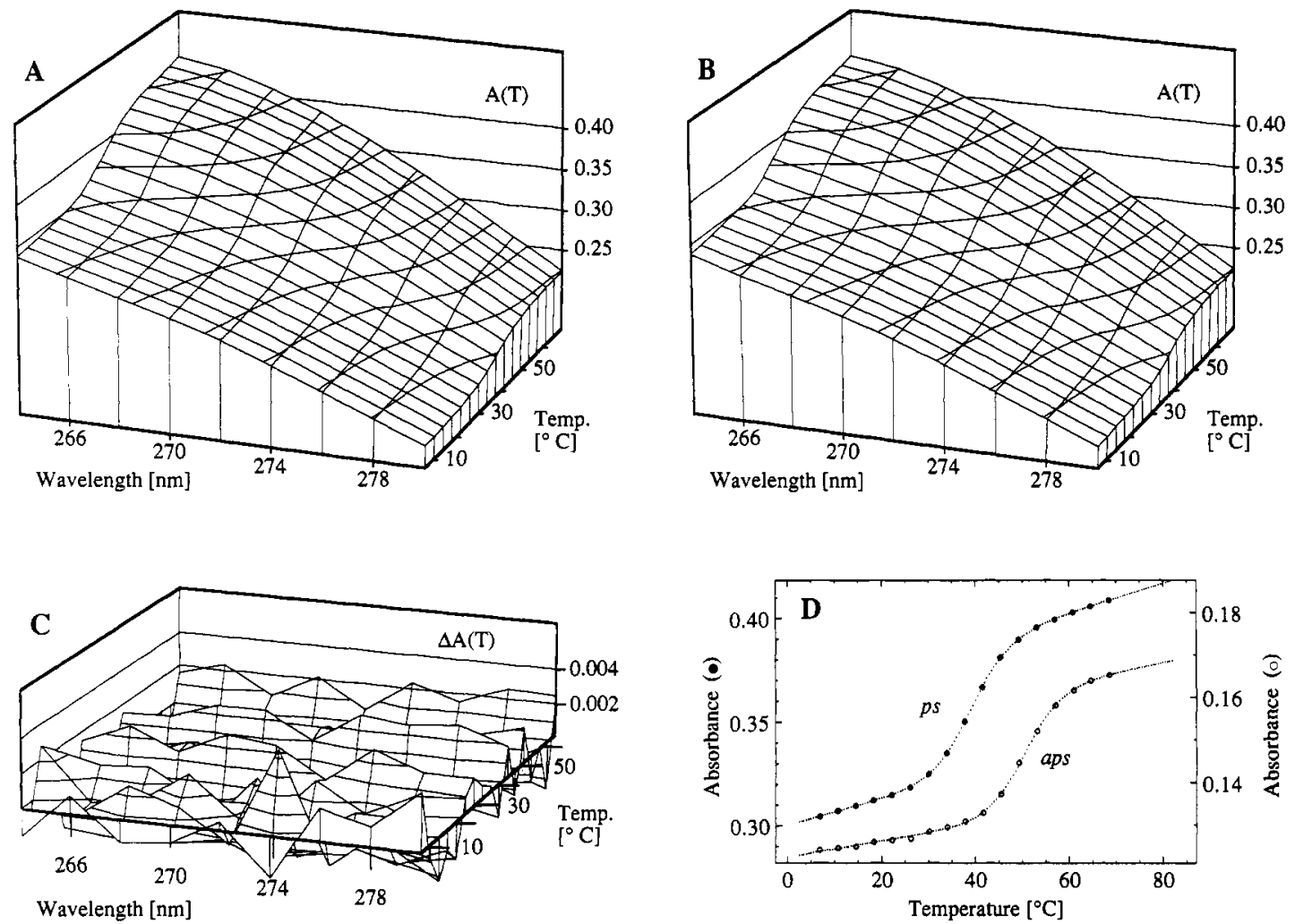

FIGURE 4: Fit to the two-state transition model of absorbance profiles for thermal denaturation of the ps-C10 hairpin in $0.1 \mathrm{M} \mathrm{NaCl}$ and 10 $\mathrm{mM}$ sodium cacodylate. (A) Experimental slices of $A_{\lambda}$ vs $T$ in region of significance (hyperchromicity $>1.3$ ). (B) Simulated surface generated from parameters derived from global analysis. (C) Difference between experimental and simulated data. Note the 25 -fold expanded scale. (D) Representative $A_{268}$ vs $T$ slices for ps-C10 (@) and aps-C10 (O): (points) experimental data; (dotted lines) simulated curves.
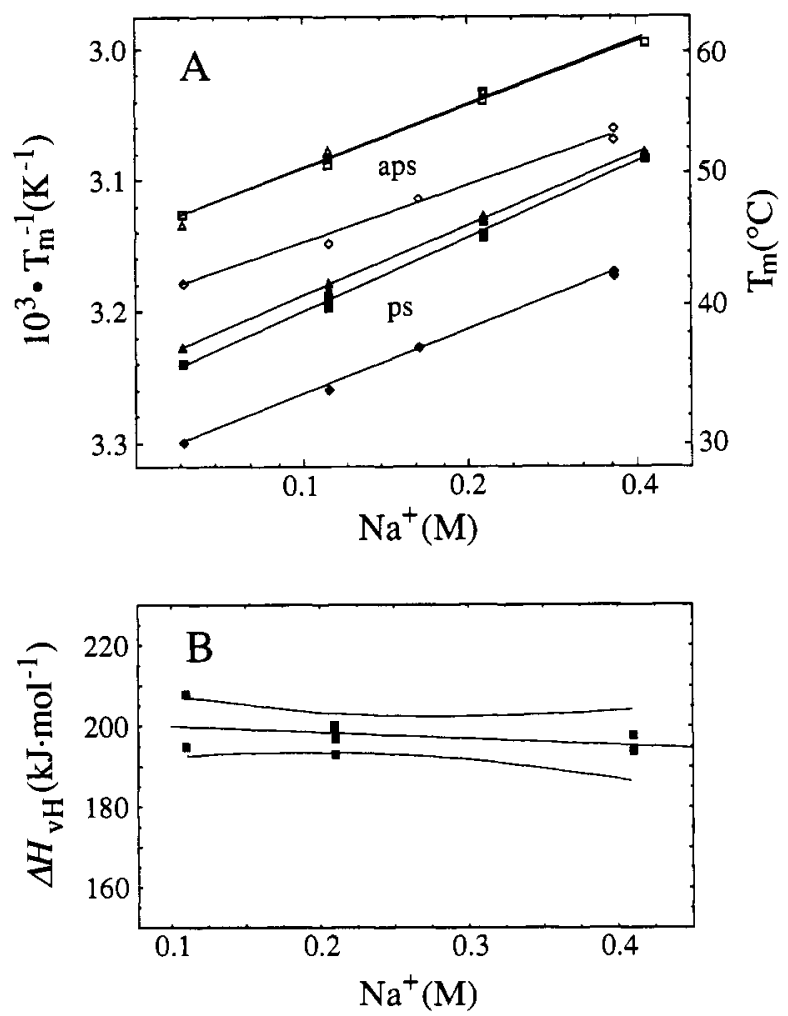

FIGURE 5: Melting temperature, $T_{\mathrm{m}}$, and van't Hoff enthalpy of melting, $\Delta H_{\mathrm{vH}}$, as function of salt concentration. The $\mathrm{Na}^{+}$concentration includes the buffer contribution. (A) Dependence of $1 / T_{\mathrm{m}}$ for the ps hairpins on $\log \left(\mathrm{Na}^{+}\right.$concentration): ps-C8 $(\diamond) ; \mathrm{ps}-\mathrm{Cl} 10$

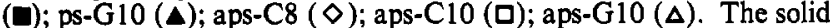
lines are linear regressions with slopes as noted in Table III. (B) Independence of $\Delta H_{v \mathrm{H}}$ for ps-C10 (D) upon $\mathrm{Na}^{+}$concentration. The solid line is the linear regression; the curved lines are the $95 \%$ confidence limits of $\Delta H_{\mathrm{yH}}$ as a function of $\mathrm{Na}^{+}$concentration.
$\mathrm{NaCl}$

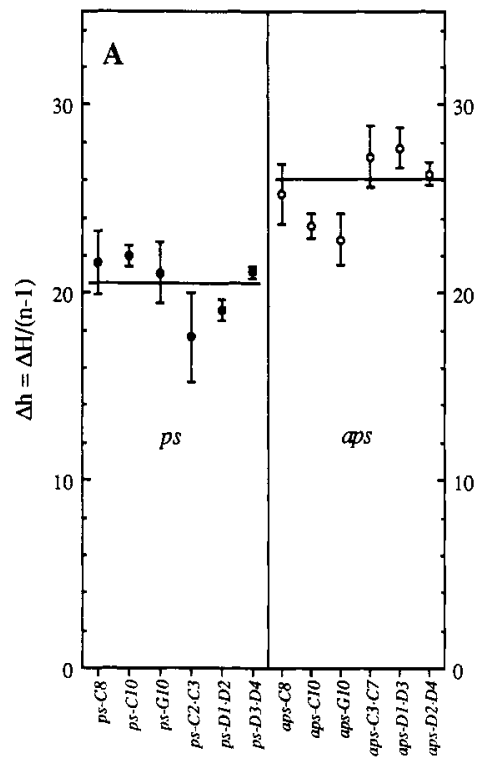

$\mathrm{MgCl}_{2}$

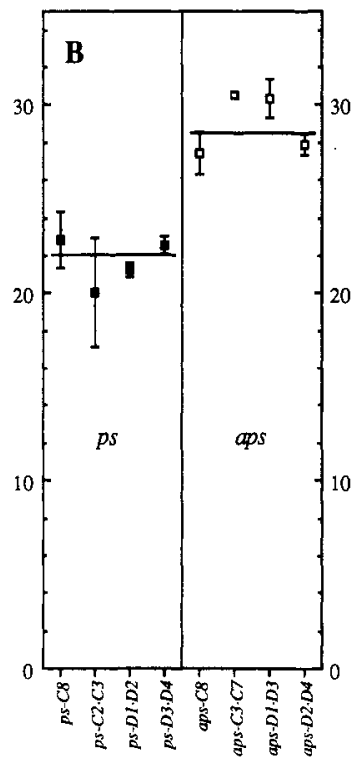

FIGURE 6: van't Hoff enthalpy of melting $\Delta h$ per nearest-neighbor interaction $(n-1)$ for $\mathrm{ps}$ and aps duplexes. Error bars are $95 \%$ confidence limits. (A) Average $\Delta h$ for measurements in $0.1-1.0 \mathrm{M}$ $\mathrm{NaCl}$ : ps (๑); aps (O). Solid lines are average values for each group of ps or aps molecules. (B) Average $\Delta h$ for measurements in 0.5-8.0

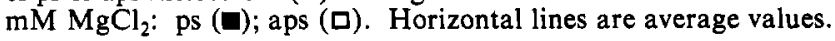

individual oligonucleotides showed significantly different interaction enthalpies $\Delta h$, with the same relative pattern for determinations in both $\mathrm{Na}^{+}$and $\mathrm{Mg}^{2+}$. We conclude, therefore, that the differences are real and ascribe them to different frequencies of the various nearest-neighbor base pairs and possibly to different nucleation contributions in the case of linear duplexes compared to the hairpin molecules. The ex- 
Table II: Thermodynamic Data for Helix-Coil Transitions of ps-DNA and aps-DNA ${ }^{a}$

\begin{tabular}{|c|c|c|c|c|c|c|}
\hline \multirow[b]{2}{*}{ DNA duplex } & \multicolumn{2}{|c|}{$\Delta H_{\mathrm{vH}}^{b}(\mathrm{~kJ} / \mathrm{mol})$} & \multicolumn{2}{|c|}{$T \Delta S^{c}\left(\mathrm{~kJ} / \mathrm{mol}, 25^{\circ} \mathrm{C}\right)$} & \multicolumn{2}{|c|}{$\Delta G^{d}\left(\mathrm{~kJ} / \mathrm{mol}, 25^{\circ} \mathrm{C}\right)$} \\
\hline & $0.1-1.0 \mathrm{M} \mathrm{NaCl}$ & $0.5-8 \mathrm{mM} \mathrm{MgCl}$ & $0.1 \mathrm{M} \mathrm{NaCl}$ & $2 \mathrm{mM} \mathrm{MgCl}$ & $0.1 \mathrm{M} \mathrm{NaCl}$ & $2 \mathrm{mM} \mathrm{MgCl}$ \\
\hline ps-C8 & $152(8)$ & $160(6)$ & $147(8)$ & $152(7)$ & $5(8)$ & $8(6)$ \\
\hline $\mathrm{ps}-\mathrm{C} 10$ & $198(2)$ & & $188(2)$ & & $10(2)$ & \\
\hline $\mathrm{ps}-\mathrm{G} 10$ & $190(8)$ & & $180(8)$ & & $10(8)$ & \\
\hline $\mathrm{ps}-\mathrm{C} 2 \cdot \mathrm{C} 3$ & $353(13)$ & $400(15)$ & $317(15)$ & $353(15)$ & $36(2)$ & $47(8)$ \\
\hline $\mathrm{ps}-\mathrm{D} 1 \cdot \mathrm{D} 2$ & $459(3)$ & $507(2)$ & $418(3)$ & $453(2)$ & $41(<1)$ & $54(<1)$ \\
\hline $\mathrm{ps}-\mathrm{D} 3 \cdot \mathrm{D} 4$ & $507(2)$ & $542(2)$ & $466(2)$ & $484(2)$ & $42(<1)$ & $58(<1)$ \\
\hline aps-C8 & $177(6)$ & $192(4)$ & $166(6)$ & $177(4)$ & $11(6)$ & $15(4)$ \\
\hline aps-C10 & $212(3)$ & & $195(3)$ & & $17(3)$ & \\
\hline aps-G10 & $206(6)$ & & $189(6)$ & & $16(6)$ & \\
\hline aps-C $3-C 7$ & $546(6)$ & $610^{e}$ & $484(6)$ & $532^{e}$ & $62(3)$ & $78^{e}$ \\
\hline aps-D1.D3 & $670(4)$ & $736(3)$ & $590(4)$ & $641(4)$ & $80(2)$ & $95(2)$ \\
\hline aps-D2.D4 & $628(2)$ & $669(2)$ & $555(2)$ & $581(2)$ & $73(1)$ & $88(1)$ \\
\hline
\end{tabular}

${ }^{a}$ The listed parameters are average values obtained from numerous experiments with $0.1-1,0 \mathrm{M} \mathrm{NaCl}$ or $0.5-8 \mathrm{mM} \mathrm{MgCl}$ and $10 \mathrm{mM}$ sodium cacodylate, $\mathrm{pH}$ 7.2. Analyses of both the increasing and decreasing temperature slopes are included. The number in parentheses beside each entry is the relative magnitude of the $95 \%$ confidence limits in percent. For example, 152 (8) signifies an average value of 152 with a $95 \%$ confidence limit of $8 \%$, that is, $\pm 12{ }^{b} \Delta H_{\mathrm{vH}}$ is the average van't Hoff enthalpy of melting. No correlation with salt concentration in the range $0.1-1.0 \mathrm{M} \mathrm{NaCl}$ or $0.5-8 \mathrm{mM} \mathrm{MgCl} 2$ was observed. ' $T \Delta S$ is the product of temperature, $T(=298.1 \mathrm{~K})$, and entropy of melting, $\Delta S$, at $25^{\circ} \mathrm{C}$ in either $0.1 \mathrm{M} \mathrm{NaCl}$ or $2 \mathrm{mM} \mathrm{MgCl}$. The entropy was calculated from eq 12 and 13 for hairpins and linear duplexes, respectively, by using the average $\Delta H_{\mathrm{vH}}$ and the linear regression estimates of $T_{\mathrm{m}}$ in $0.1 \mathrm{M} \mathrm{NaCl}$ and $2 \mathrm{mM} \mathrm{MgCl}_{2}$ from Table III. ${ }^{d} \Delta G$ is the average Gibbs free energy of transition calculated from eq 11 by using the average $\Delta H_{\mathrm{vH}}$ and the estimate of $T \Delta S$. 'Too few measurements were made to calculate $95 \%$ confidence limits of the average.

Table III: Salt Dependence of Melting Temperatures ${ }^{a}$

\begin{tabular}{|c|c|c|c|c|c|c|c|c|}
\hline \multirow[b]{4}{*}{ DNA duplex } & \multirow{2}{*}{\multicolumn{2}{|c|}{$T_{\mathrm{m}}^{b}\left({ }^{\circ} \mathrm{C}\right)$}} & \multicolumn{4}{|c|}{$1 / T_{\mathrm{m}}=\alpha+\beta \log c^{c}$} & & \\
\hline & & & \multicolumn{2}{|c|}{$\mathrm{NaCl}(\mathrm{M})$} & \multicolumn{2}{|c|}{$\mathrm{MgCl}_{2}(\mathrm{mM})$} & \multirow{2}{*}{\multicolumn{2}{|c|}{$\partial T_{\mathrm{m}} / \partial \log c^{d}\left({ }^{\circ} \mathrm{C}\right)$}} \\
\hline & $0.1 \mathrm{M}$ & $2 \mathrm{mM}$ & $\alpha \times 10^{3}$ & $\beta \times 10^{3}$ & $\alpha \times 10^{3}$ & $\beta \times 10^{3}$ & & \\
\hline & $\mathrm{NaCl}$ & $\mathrm{MgCl}_{2}$ & $\left(K^{-1} \times 10^{3}\right)$ & $\left(K^{-1} \times 10^{3}\right)$ & $\left(K^{-1} \times 10^{3}\right)$ & $\left(K^{-1} \times 10^{3}\right)$ & $\mathrm{NaCl}$ & $\mathrm{MgCl}_{2}$ \\
\hline \multicolumn{9}{|c|}{ parallel-stranded duplexes } \\
\hline $\mathrm{ps} \cdot \mathrm{C} 8$ & $33.5(2)$ & $41.0^{e}$ & $3.10(<1)$ & $-0.17(8)$ & & & $16(9)$ & \\
\hline $\mathrm{ps} \cdot \mathrm{C} 10$ & $39.4(1)$ & & $3.01(<1)$ & $-0.19(7)$ & & & $19(8)$ & \\
\hline $\mathrm{ps}-\mathrm{G} 10$ & $40.6(1)$ & & $3.01(<1)$ & $-0.18(5)$ & & & $18(6)$ & \\
\hline $\mathrm{ps}-\mathrm{C} 2 \cdot \mathrm{C} 3$ & 25.1 (4) & $33.8^{e}$ & $3.19(<1)$ & $-0.17(13)$ & & & $15(12)$ & \\
\hline $\mathrm{ps}-\mathrm{D} 1 \cdot \mathrm{D} 2$ & $28.5(4)$ & $35.7^{e}$ & $3.11(<1)$ & $-0.21(12)$ & & & $17(10)$ & $7^{e}$ \\
\hline ps-D3.D4 & $28.4(5)$ & $37.4(2)$ & $3.11(<1)$ & $-0.22(17)$ & $3.26(<1)$ & $-0.10(12)$ & $19(14)$ & $10(11)$ \\
\hline \multicolumn{9}{|c|}{$\begin{array}{l}\text { antiparallel-stranded duplexes } \\
\text { a }\end{array}$} \\
\hline aps-C8 & $44.7(2)$ & $49.7^{e}$ & $3.00(<1)$ & $-0.15(17)$ & & & $15(18)$ & \\
\hline aps-C10 & $50.4(1)$ & & $2.93(<1)$ & $-0.16(11)$ & & & $17(11)$ & \\
\hline aps-G10 & $50.5(2)$ & & $2.93(<1)$ & $-0.16(19)$ & & & $17(17)$ & \\
\hline aps-C $3 . C_{7}$ & $40.0(2)$ & $46.8^{e}$ & $3.04(<1)$ & $-0.16(11)$ & & & $16(9)$ & \\
\hline aps-D 1.D3 & $46.0(2)$ & $50.8^{e}$ & $2.97(<1)$ & $-0.17(10)$ & & & $16(8)$ & $5^{e}$ \\
\hline aps-D2.D4 & $43.5(1)$ & $50.3(2)$ & $2.99(<1)$ & $-0.17(7)$ & $3.12(<1)$ & $-0.09(11)$ & $17(6)$ & $8(9)$ \\
\hline
\end{tabular}

${ }^{a}$ The listed parameters are obtained from a linear regression of $1 / T_{\mathrm{m}}$ and $T_{\mathrm{m}}$ versus $\log c$, where $c$ is the salt concentration, of observations with $0.05-0.5 \mathrm{M} \mathrm{NaCl}$ or with $0.12-2 \mathrm{mM} \mathrm{MgCl}{ }_{2}$ and $10 \mathrm{mM}$ sodium cacodylate, $\mathrm{pH} 7.2$. The experimental $T_{\mathrm{m}}$ of measurements with linear duplexes having a total strand concentration other than $2 \mu \mathrm{M}$ were adjusted according to

$$
\frac{1}{T_{\mathrm{m}}{ }^{\text {cor }}}=\frac{R}{\Delta H_{\mathrm{vH}}} \ln \left(\frac{C_{\mathrm{t}}^{\text {obs }}}{2}\right)+\frac{1}{T_{\mathrm{m}}^{\text {obs }}}
$$

derived from eq 14, where $C_{\mathrm{i}}$ obs is the observed strand concentration (micromolar), $T_{\mathrm{m}}$ obs the observed melting temperature, and $T_{\mathrm{m}}{ }^{\text {cor }}$ the corrected melting temperature. Only results derived from data for increasing temperatures were included in the regressions. The number in parentheses beside each entry is the relative magnitude of the $95 \%$ confidence limits in percent. For example, 33.5 (2) signifies an average value of 33.5 with a $95 \%$ confidence limit of $2 \%$, that is, $\pm 0.7 .{ }^{b} T_{\mathrm{m}}$ is the melting temperature in $0.1 \mathrm{M} \mathrm{NaCl}$ or $2 \mathrm{mM} \mathrm{MgCl} 2$ with a total strand concentration of $2 \mu \mathrm{M}$. It is estimated from the linear regression of $1 / T_{\mathrm{m}}$ versus $\log c .{ }^{c}$ Linear regression coefficients for $1 / T_{\mathrm{m}}$ as a function of $\log c$. $\alpha$ is the intercept and $\beta$ the slope of the regression line. ${ }^{d}$ The slope of a linear regression of $T_{\mathrm{m}}$ versus $\log c$. ${ }^{e}$ Too few measurements were made to calculate $95 \%$ confidence limits of the average.

tensive sequence similarities, however, do not permit accurate estimation of the specific enthalpic contributions from the various dinucleotides. The overall average values (horizontal lines in Figure 6) are as follows: (1) for $\mathrm{NaCl}$ ps-DNA 20 $\mathrm{kJ} \mathrm{mol}^{-1}$, aps-DNA $26 \mathrm{~kJ} \mathrm{~mol}^{-1}$; and (2) for $\mathrm{MgCl}_{2}$ ps-DNA $22 \mathrm{~kJ} \mathrm{~mol}^{-1}$, aps-DNA $29 \mathrm{~kJ} \mathrm{~mol}^{-1}$. The marked stabilization due to $\mathrm{Mg}^{2+}$ mentioned before results in a higher cooperativity of the transition and hence increased values for $\Delta h$. The observed enthalpy changes per mole of nearest-neighbor interaction for the aps-DNAs are somewhat lower (ca. 20\%) than those previously reported by Breslauer et al. (1986), i.e., $38 \mathrm{~kJ} \mathrm{~mol}^{-1}$ for a d(AA) $\cdot \mathrm{d}(\mathrm{TT})$ dinucleotide, $36 \mathrm{~kJ} \mathrm{~mol}^{-1}$ for $\mathrm{d}(\mathrm{AT}) \cdot \mathrm{d}(\mathrm{TA})$, and $25 \mathrm{~kJ} \mathrm{~mol}^{-1}$ for $\mathrm{d}(\mathrm{TA}) \cdot \mathrm{d}(\mathrm{AT})$. The ob- served discrepancy may be in part due to fraying of the ends, which would decrease the apparent cooperativity of the transition and hence $\Delta H_{\mathrm{vH}}$ [Breslauer et al. (1986) incorporated $\mathrm{G} \cdot \mathrm{C}$ base pairs at the ends of their oligonucleotide reference molecules].

The $T_{\mathrm{m}}$ values of both the ps and aps duplexes reveal a classical dependence on salt concentration. Figure 5A shows the relation $T_{\mathrm{m}}{ }^{-1}$ versus $\log \left(\left[\mathrm{Na}^{+}\right]\right)$for the various hairpins. [Data for the linear duplexes are in Ramsing and Jovin (1988), Figure 9, and Rippe et al. (1989), Figure 7.] The relationships are approximately linear for $\mathrm{NaCl}$ concentration in the range 0.05-0.5 M, but not above (Rippe et al., 1989). Regression coefficients of all the examined duplexes are listed in Table 
III, together with the estimates of $T_{\mathrm{m}}$ in $0.1 \mathrm{M} \mathrm{NaCl}$ obtained from these lines. Transition temperatures in $\mathrm{MgCl}_{2}$, where available, are also included in Table III.

At any given $\mathrm{NaCl}$ concentration, the melting temperatures of the ps hairpins were $10-11^{\circ} \mathrm{C}$ lower than that of the corresponding aps hairpins, as compared to the $13-18^{\circ} \mathrm{C}$ difference observed with the linear duplexes (Ramsing \& Jovin, 1988; Rippe et al., 1989). The slope $\partial\left[T_{\mathrm{m}}^{-1}\right] / \partial \log \left[\mathrm{Na}^{+}\right]$was constant in the range $0.05-0.5 \mathrm{M} \mathrm{NaCl}$ and appeared slightly higher for the ps-DNA than for the aps-DNA, the average values being $-0.18 \times 10^{-3}$ and $-0.16 \times 10^{-3}$, respectively. The values are comparable, however, suggesting that the thermodynamic degree of ion dissociation accompanying denaturation is similar for all species (Record et al., 1978; Manning, 1978). Another common measure of the salt dependence is the change of $T_{\mathrm{m}}$ per decade of salt concentration, $\partial T_{\mathrm{m}} / \partial \log \left[\mathrm{Na}^{+}\right]$, which was $15-19^{\circ} \mathrm{C}$ for all species examined and comparable to the value of $\sim 20{ }^{\circ} \mathrm{C}$ reported for natural sequences of DNA (Record, 1975). We conclude from the above discussion and the $\Delta G$ estimates for $25^{\circ} \mathrm{C}$ (Table II) that even short linear ps duplexes are stable under physiological conditions, particularly in the presence of $\mathrm{Mg}^{2+}$ (and presumably other divalent cations), a fortunate circumstance for enzymatic and other biochemical studies with this form of DNA (Rippe \& Jovin, 1989).

Conclusions. This study establishes that the thermodynamic properties (helical stabilities) of parallel-stranded DNA duplexes formed by hybridization of conventional strands (Ramsing \& Jovin, 1988; Rippe et al., 1989) are virtually identical with those of hairpin constructs (van de Sande et al., 1988). It is important to stress that in the ps, aps comparisons we are not dealing with equilibria between these alternative helical forms. Rather, ps-DNA is to be regarded as the natural double-helical structure that forms between two strands with sequence complementarity in the parallel orientation. It is important to determine whether G.C base pairs are compatible with the architecture of ps-DNA. We will report elsewhere the thermodynamic consequences of interspersing G.C base pairs in the D series of oligonucleotides. Other investigations are required to establish whether (1) the sequence motifs identified as potential loci for ps-DNA in vivo (Ramsing \& Jovin, 1988) actually exist and (2) proteins specific for psDNA can be identified. Although only refined structural analysis will confirm the basic structural features of ps-DNA, theoretical calculations (A. Garcia, D. M. Soumpasis, and T. M. Jovin, unpublished data) are in agreement with the thermodynamic properties of the ps helix derived from experiment.

\section{ACKNOWLEDGMENTS}

We are indebted to Karl-Herman Fuchs for setting up the computer control of the Uvikon spectrophotometer and to Gudrun Heim for technical assistance.

\section{REFERENCES}

Breslauer, K. J., Frank, R., Blöcker, H., \& Marky, L. A. (1986) Proc. Natl. Acad. Sci. U.S.A. 83, 3746-3750.

Germann, M. W., Pon, R. T., \& van de Sande, J. H. (1987) Anal. Biochem. 165, 399-407.

Germann, M. W., Kalish, B. W., \& van de Sande, J. H. (1988) Biochemistry 27, 8302-8306.

Manning, G. S. (1978) Q. Rev. Biophys. 11, 179-246.

Marky, L. A., \& Breslauer, K. J. (1987) Biopolymers 26, 1601-1620.

Pattabiraman, N. (1986) Biopolymers 25, 1603-1606.

Ramsing, N. B., \& Jovin, T. M. (1988) Nucleic Acids Res. $16,6659-6676$.

Record, M. T., Jr. (1975) Biopolymers 14, 2137-2158.

Record, M. T., Jr., Anderson, C. F., \& Lohman, T. M. (1978) Q. Rev. Biophys. 11, 103-178.

Rippe, K., \& Jovin, T. M. (1989) Biochemistry (third of three papers in this issue).

Rippe, K., Ramsing, N. B., \& Jovin, T. M. (1989) Biochemistry (second of three papers in this issue).

van de Sande, J. H., Ramsing, N. B., Germann, M. W., Elhorst, W., Kalisch, B. W., v. Kitzing, E., Pon, R. T., Clegg, R. M., \& Jovin, T. M. (1988) Science 241, 551-557. 